\title{
Automatic Weed Detection and Smart Herbicide Sprayer Robot
}

\author{
G.Y. Rajaa Vikhram ${ }^{1 *}$, Rakshit Agarwal ${ }^{2}$, Rohan Uprety ${ }^{3}$, V.N.S. Prasanth ${ }^{4}$ \\ ${ }^{1}$ Electronics And Instrumentation, SRM University, Chennai, India. \\ ${ }^{2}$ Electronics And Instrumentation, SRM University, Chennai, India. \\ E-Mail:Rakshitagarwal1996@Gmail.Com \\ ${ }^{3}$ Electronics And Instrumentation, SRM University, Chennai, India. \\ E-Mail:Upretyrohan@Gmail.Com \\ ${ }^{4}$ Electronics And Instrumentation, SRM University, Chennai, India. \\ E-Mail:Vnsaiprasanth@Gmail.Com \\ *Corresponding Author E-Mail:Rajaavikhram.Y@Ktr.Srmuniv.Ac.In
}

\begin{abstract}
The ordinary method for murdering weeds (unwanted plants) in a harvest manor is to shower herbicides all over the estate. This outcomes in defilement of the sustenance crops and furthermore the yield turns out to be less as a portion of the production plants pass on alongside the weeds. In this way, there is a requirement for a brilliant weed control framework. In this venture, a picture handling calculation is utilized to take pictures of the manor columns at consistent interims and after recognizing the weeds in the captured image, the weed killer chemical is showered specifically and just on the weeds. The herbicide is put away in a compartment fitted with water pump engines joined to shower spouts. After the weeds are recognized, a flag is signaled from Raspberry Pi to the motor driver IC governing the water pump motors to shower the chemicals over the unwanted vegetation.
\end{abstract}

Keywords: RaspberryPi, weed, python, herbicides, image processing.

\section{Introduction}

Cultivation is the establishment of Indian economy. It is a decent spring of regular payment concerning half of the Indian populace. It is principally create arranged and thus the benefit or misfortune sustained on the yield got. One of the significant effects in agribusiness is the control of weeds developing among the estate crops. At the exhibit, these sorts of plants are being expelled physically, in whatever place conceivable, or herbicides are being splashed consistently everywhere throughout the field to hold them in check. In customary weed control frameworks, herbicides are splashed consistently all over the field. This procedure is incompetent as pretty much $20 \%$ of the shower achieves the plant and under $1 \%$ of the synthetic really adds to weed control, prompting wastage, sullying of nature and wellbeing issues in individuals [1]. To maintain a strategic distance from these outcomes, a keen weed control framework ought to be utilized. These frameworks must be equipped for finding weeds in the field and herbicide sprayers are coordinated to splash ideal on the coveted specks. Additionally, it centres on decreasing the expensive work and limits the utilization of herbicides that damage the ordinary development of plants. The machine vision keystone way utilizes surface, shape, shading and area-based highlights exclusively or in a mix of these to separate amongst unwanted plant and product. An imaging detector is a main part of any weed location and arrangement framework. Singular plant characterization has been effectively shown with either otherworldly or shading imaging. The spatial resolutions of phantom frameworks are commonly not sufficient for exact person plant or leaf identification. Weed regulation action is a basic problem and can essentially influence edit yield. Herbicides assume a vital part of weed control in any case, their utilization is condemned on the grounds that it is utilized exorbitantly and has possibly hurtful impacts. Numerous examinations show that utilization of herbicides is lessened by fix showering. Manual exploring for fix showering requires extensive assets and isn't an attainable alternative [2]. Numerous scientist has examined Patch splashing utilizing remote detecting and machine vision. Machine vision frameworks are appropriate for plant scale though remote detecting can be utilized on idea proposed. Both of these frameworks basically need picture procurement and picture handling. Picture estimate goes in the request of megabytes, in this manner preparing time contingent upon picture determination, yield and weed compose, calculation utilized and equipment designs. The initial phase of recognizing weeds inside a picture includes grouping the pixels. The motivation behind portioning the picture into the plant and foundation pixels is to recognize the sum of plant material inside a particular zone. In the event that the measure of plant material achieves a particular limit, that territory is focused on herbicidal spray application. A framework that could make utilization of the spatial appropriation data continuously and apply as it were the fundamental measures of herbicide to the weed-invaded region would be significantly more effective and limit natural harm. In this way, a high spatial determination, ongoing weed invasion recognition framework is by all accounts the answer for site-specific weed administration.

\section{Methodology Adopted}

The working of the framework comprises of three principle steps. Fig. 1 demonstrates the procedure stream of the calculation.

- The underlying advance is picture securing that is refined by the Raspberry-Pi Camera. The camera is mounted 
facing earthwards on an expanded segment arm from the occasion of the robot at a stature of around 40 centimeters from the land. Picture acquisition is done inside seeing trademark light.

- The accompanying advance is the preparing of the caught picture. The picture is enslaved to morphological thresholding, erosion and dilation to decide the nearness of yields in the Region of Interest recognizing whether it is a weed or the plant [3].

- The definitive advance is the straightway splash of the weeds in the ROI.

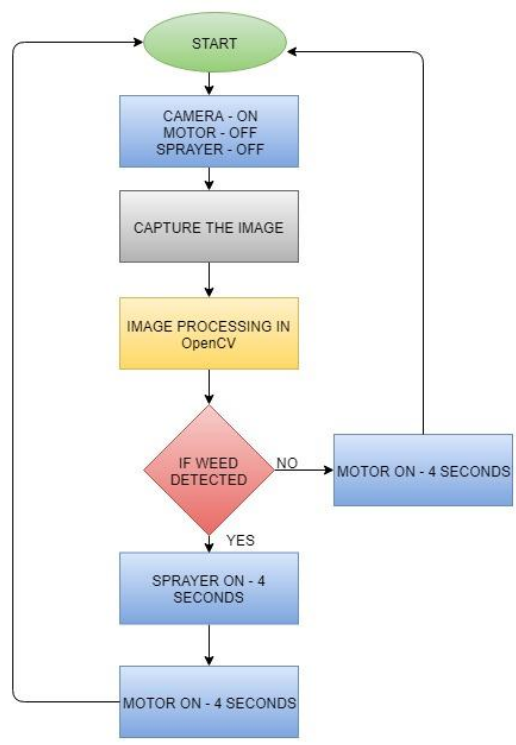

Fig. 1: Process Flow chart

\section{Algorithm for Weed Detection}

The process set about by capturing the image form Raspberry-Pi Camera. The image is then converted to grayscale using cv2.cvtColor() of OpenCV. Then we mask image to green colour by giving masking range of $(36,0,0)(86,255,255)$ in cv2.inRange() function. After doing this we only get green part of the image. Then the image will by default be converted to black and white image where black pixels represent background image and white pixels represent green image [4]. We calculate threshold value by giving multiple weed images. For the weed, the threshold value will be more because of broad leaves. So we set a condition, that if the threshold value of input image is greater than the set threshold value, our image is weed image, else there is no weed in the image [5]. The threshold value here taken is around 90000.

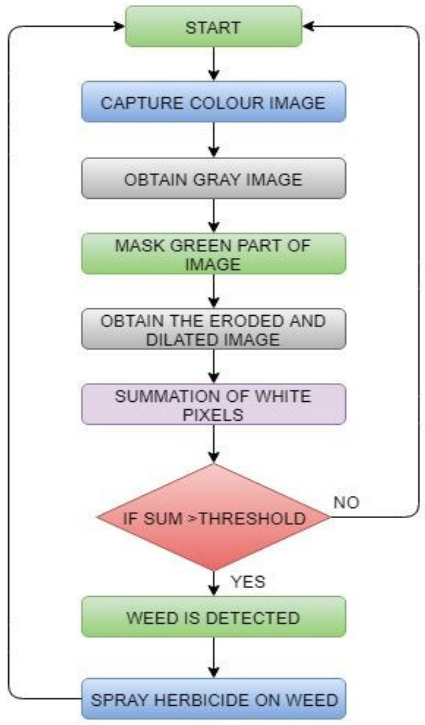

Fig. 2: Flowchart of the algorithm

\section{Spraying on the Weeds}

There are 3 separate codes for wheel motor, detection of weeds, and pump motor. Basically, there are three different python scripts. So all these 3 python scripts are imported in the fourth python script. We only run the fourth python script. In the script, we have given a condition, that if weed is detected then spray motor will be on and if weed is not detected then wheel motor is on. This condition is put in a while loop, such that this process will run, unless and until the user manually closes the program. The pump motor is programmed only for 4 seconds. It will spray the herbicide on the plant for 4 seconds only and then it will stop.

\section{Hardware Implementation}

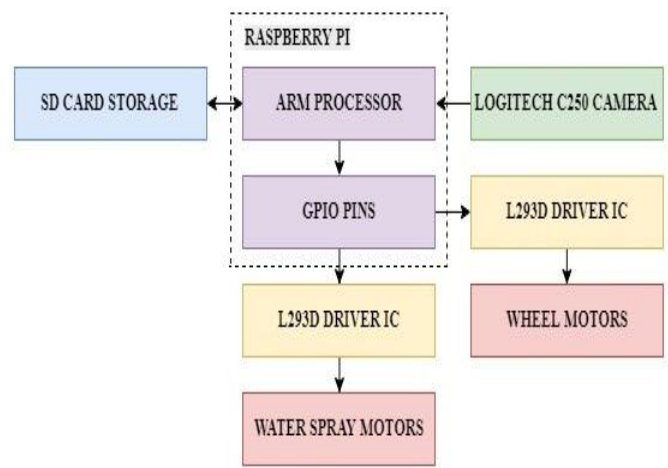

Fig. 3: Block diagram

The square chart of the system is depicted in Figure 3. The gear fuses an able ARM processor $\mathrm{Pi}$ controller. The previously mentioned algorithmic govern is composed utilizing Python content on the Raspberry-Pi. Pi USB Camera is used to catch the photographs of the harvests. The photos thusly captured are processed, and a decision is gone up against the premise of a computation of white pixels in the photo and the herbicide is showered. The showering is master by the usage of water shower motors pipe concentrating clearly on the plants. These portions are mounted on a land robot that movements through the ground, catching pictures at predefined between times and taking care of each photo taken, and in this way sprinkling the herbicide.

A motor driver L293D IC is utilized for interfacing the wheel motors and pump motors with Raspberry-Pi. The wheels of the robot are connected to equipped $30 \mathrm{rpm}$ engines initiated by a $9 \mathrm{~V}$ battery. The robot holds one little pump motor kept in holder accused of herbicide, joined to a pipe for splashing.. Robot Dimensions

- Chassis Width: $-30 \mathrm{~cm}$

- Chassis Length: $-23 \mathrm{~cm}$

- $\quad$ Front Wheels Width: $-1.7 \mathrm{~cm}$

- Rear Wheels Width: $-3.5 \mathrm{~cm}$

- Wheelbase: $-19 \mathrm{~cm}$

- L-shaped Column: - 40x25 cm

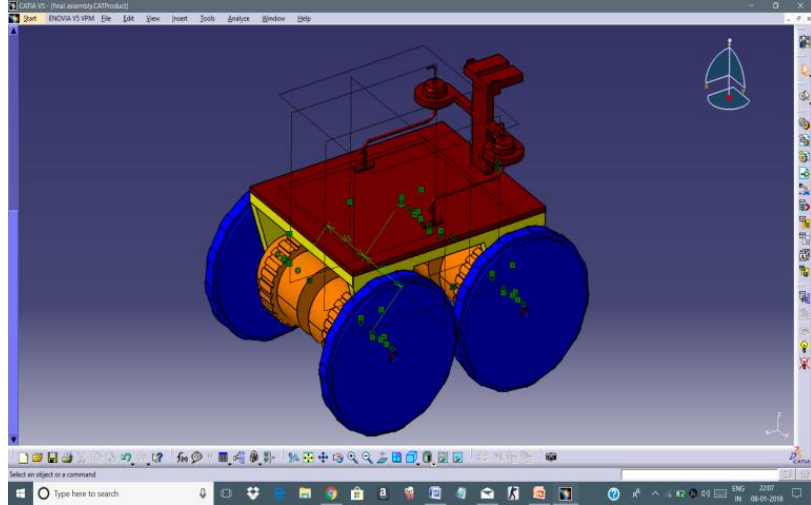

Fig. 4: CAD model 


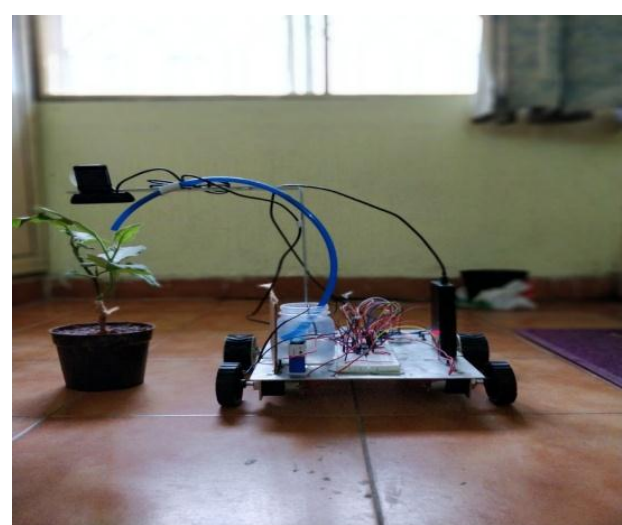

Fig. 5: Developed robot

The Smart Robot delineates the total framework configuration appeared in Fig 5.

The wheel motors and water pump motors are consolidated to the Raspberry-Pi through L293D IC driver. The Pi is enacted by the $5 \mathrm{~V}$ control supply. The camera is interconnected to USB Port of Raspberry Pi.

The driver circuit of the front wheel engines are controlled by the GPIO Pins 16, 18, 22 for the back wheel 23, 21, 19 and the driver circuit controlling the water pump motor are associated with GPIO Pins 36, 38 and 40.

For Raspberry-pi

1) Memory card of $16 \mathrm{~GB}$ or $32 \mathrm{~GB}$

2) HDMI monitor for displaying.

3) Ethernet cable for providing web access.

4) Keyboard and Mouse.

5) 5-Volt supply.

6) Sensors.

Software Used:

Programming Language: -Python

O.S:-Raspbian

\section{Technologies Used}

\section{A. Python}

Python is a widely employed universally useful, abnormal state programming language. Its vogue rationality stresses code meaningfulness, and its punctuation enables software masterminds to exact thoughts in fewer lines of code that may be achievable in language, for example, Java or $\mathrm{C}++$. The language gives relevant develops which should empower clearing programs on both a little and far-reaching scale. Python aides and supports various programming ideal models, moreover as question arranged, basic and handy programming or procedural styles. It has colossal features of dynamic sort framework and customized memory administration and containing a goliath and standard library.

Github link to our source code:

https://github.com/rakshitagarwal96/Automatic-Weed-Detection

\section{B. OpenCV}

OpenCV (Open Source Computer Vision Library) is an open source PC vision and machine learning programming library. OpenCV was worked to give a typical foundation to PC vision applications and to quicken the utilization of machine observation in the business items. Being a BSD-authorized item, OpenCV makes it simple and easy for organizations to use and conform the code. The library has in excess of 2500 enhanced and estimated calculations, which joinsa far-reaching set of both exceptional and best in class PC vision and machine learning calculations. These calculations can be utilized to distinguish and perceive faces, distinguish objects, characterize human activities in recordings, track camera developments, track moving items, separate 3D models of articles, create 3D point mists from stereo cameras, fasten pictures together to deliver a high determination picture of a whole scene, find comparable pictures from a picture database, remove red eyes from pictures taken employing streak, take after eye improvements, discern landscape and set up markers to superimpose it with broadened reality, and so on. OpenCV has in excess of 47 thousand individuals of client group and evaluated a number of downloads surpassing 14 million. The library is utilized widely in organizations, inquire about gatherings and by legislative bodies.

\section{Simulation Results}

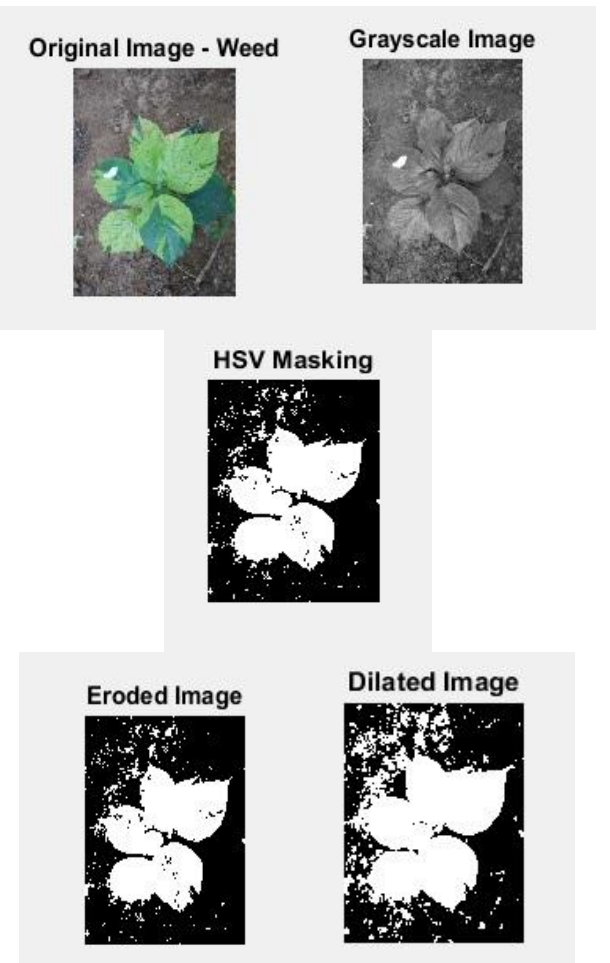

Fig. 6: Different stages of image processing of weed plant

\section{Conclusion}

A calculation to recognize weeds among estates was effectively created and a model of the Automatic Weed Detection and Smart Herbicide Sprayer Robot was outlined and actualized effectively. The robot involved equipment that can bolster and adequately splash herbicides on the identified weeds progressively. A sample course was planned with a column of Ragi (E.coracana) plants also, arbitrarily set expansive leaved weeds. The weeds were effectively recognized and showered against by the robot and this procedure takes roughly 4 seconds. All the weed plants were distinguished legitimately, with a couple of Ragi (E.coracana) plants being recognized as weeds in situations where they developed in vast groups. The picture examination for weed recognition can be further enhanced by separating the picture into more number of regions and have more number of spouts to splash the chemicals. It can be transformed into an exceptionally closedloop framework by fusing a memory module for storing information. The image handling algorithm can be modified further, with the goal that the recognition turns out to be more non-specific.

\section{References}

[1] Kamarul HG, Mohd MM \&Aini H, "Machine Vision System for Automatic Weeding Strategy using Image Processing Technique", American-Eurasian J. of Agriculture\& Environmental Science, Vol.3, No.3, (2008), pp.451-458. 
[2] Amir HKB \& Ali MS, "Automatic Weed Detection System and Smart Herbicide Sprayer Robot for corn fields", IEEE Int. Conf. on Robotics and Mechatronics, Tehran, (2013), pp.468-473.

[3] Muhammad HS, Irshad A \& Suziah BS, "Weed Recognition Based on Erosion and Dilation Segmentation Algorithm", Int. Conf. on Education Technology and Computer, Singapore, (2009), pp.224-228.

[4] Rafael CG \& Richard EW, Morphological Image Processing, Digital Image Processing, 3rd edition, New Jersey, Prentice-Hall Inc., (2006), pp.649-657.

[5] Ishak AJ, Mokri SS, Mustafa MM \& Hussain A, "Weed detection utilizing quadratic polynomial and ROI techniques", IEEE 5th Student Conference on Research and Development, (2007), pp.1-5. 